\title{
VIRUS-W A fiber based integral field unit spectrograph for the study of galaxy bulges
}

\author{
Maximilian H. Fabricius ${ }^{1,2} \dagger$, Ralf Bender ${ }^{1,2}$, Niv Drory ${ }^{1,2}$, \\ Frank Grupp ${ }^{1}$, Gary J. Hill ${ }^{3}$ and Ulrich Hopp ${ }^{1,2}$ \\ ${ }^{1}$ University Observatory of the Ludwig-Maximilians University (LMU), Munich, Germany \\ ${ }^{2}$ Max-Planck Institute for Extraterrestrial Physics (MPE), Munich, Germany \\ ${ }^{3} \mathrm{McD}$ onald Observatory and Department of Astronomy, University of Texas at Austin, Austin, \\ Texas, USA
}

\begin{abstract}
We presented the design for a fiber based integral field unit spectrograph for the new two meter class Wendelstein telescope in Bavaria, Germany. The proposed spectrograph will feature a fiberhead consisting of 246 individual optical fibers and a field of view of approximately $1^{\prime} \times 2^{\prime}$ and two different spectral resolution modes optimized for the study of bulges of local latetype galaxies.
\end{abstract}

Keywords. galaxies: bulges, galaxies: kinematics and dynamics, instrumentation: spectrographs

\section{Introduction}

We develop a fiber based integral field unit (IFU) spectrograph for the new two meter Wendelstein telescope in Bavaria. The design is heavily based on the VIRUS spectrograph proposed for the HETDEX experiment (see Hill et al. 2006 to Kelz et al. 2006). It will feature a fiberhead consisting of 246 individual optical fibers and a large field of view of about $150^{\prime \prime} \times 75^{\prime \prime}$. We aim for two different modes of spectral resolution. The low resolution mode - dedicated to stellar population studies — with $\mathrm{R} \simeq 3000$ will cover the wavelength range from $4750 \AA$ to $5600 \AA$ enabling the observation of $H_{\beta}$ and the Fe5270, Fe5335 and Fe5406 lines and their corresponding pseudo-continua up to $\mathrm{z} \simeq 0.03$. The medium resolution mode - dedicated to kinematical studies - with $\mathrm{R} \simeq 7850$ will enable the study of velocity dispersions down to $17 \mathrm{~km} / \mathrm{s}$. The covered wavelength range will be $370 \AA$ wide and reach from $5070 \AA$ to $5445 \AA$. This covers the $\mathrm{Mg}$ lines at $5167 \AA, 5172 \AA$ and $5183 \AA$ up to a redshift of 0.03 . Given an about eight times larger spatial coverage and a spectral resolution which is higher by a factor of two, VIRUSW will extend studies of the SAURON (see e.g. Davies et al. 2001, Bacon et al. 2001) spectrograph into the direction of local late-type and lower mass galaxies. Based on the experience from the VIRUS prototype we estimate about one year for the construction. Since the Wendelstein $2 \mathrm{~m}$ telescope will not be available by then we may start observing with VIRUS-W at the McDonald $2.7 \mathrm{~m}$ Harlan Smith Telescope in Texas.

\section{Optical design}

The input focal ratio of the spectrograph is $\mathrm{f} / 3.35$. The fibers are arrayed in a pseudoslit which is located within a folding mirror. An anti reflective coated cylindrical normalizer lens is attached to the fibers. The two volume phase holographic gratings can

$\dagger$ Present address: Max Planck Institute for Extraterrestrial Physics (MPE), Giessenbachstrasse, D-85748 Garching, Germany 

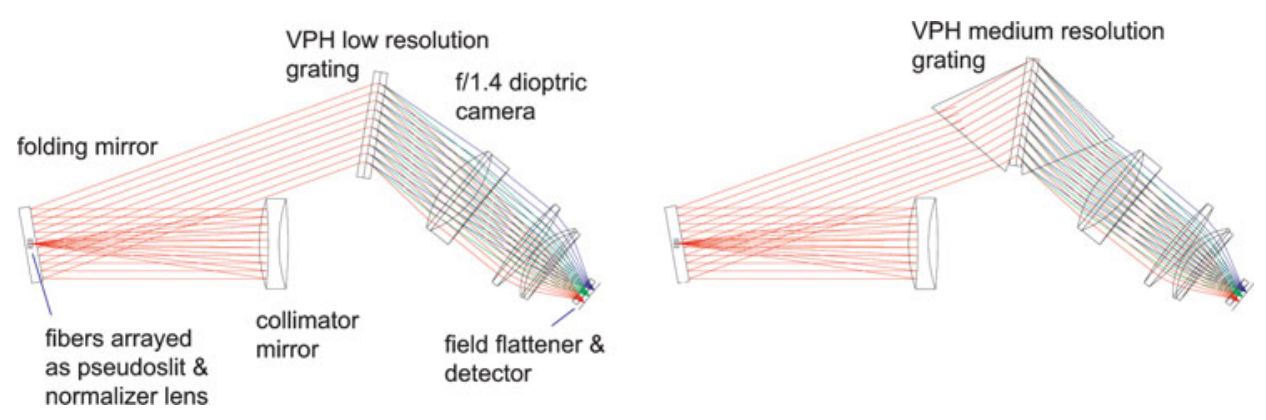

Figure 1. VIRUS-W in low resolution (left) and medium resolution (right) configuration.

be exchanged while keeping position of the camera fixed. The $\mathrm{f} / 1.4$ dioptric camera is based on the SALT telescope High Resolution Spectrograph red arm camera designed by Bernard Delabre at ESO and incorporates mostly spherical components. The utilization of fibers allows to place the spectrograph inside a climatized room underneath the telescope dome. Drifts due to temperature changes or changing $g$ vector are therefore avoided. We chose to implement a rectangular IFU geometry $20 / 21 \times 12$ fibers with a pitch of $255 \mu \mathrm{m}$. Given a f/3.65 beam this results in a head size of $5.36 \mathrm{~mm} \times 2.68 \mathrm{~mm}$ or a $150^{\prime \prime} \times 75^{\prime \prime}$ field of view, the fiber have a diameter of $4.4^{\prime \prime}$ on sky and cover an area of $14.1 \operatorname{arcsec}^{2}$ each.

\section{Sensitivity}

Based on theoretical predictions by the grating manufacturers and the current telescope design a preliminary calculation suggests a signal to noise ratio of about 10 for the medium resolution mode, a one hour exposure and an object with a $\mathrm{V}$ band surface brightness of $22 \mathrm{mag} \operatorname{arcsec}^{-2}$.

\section{Acknowledgements}

The material in this document is based on many discussions with Claus Gössl, Florian Lang, Phillip J. MacQueen and Wolfgang Mitsch. Stuart Barnes adopted the design for the SALT HRS camera for the VIRUS spectrograph. Hans Decker (ESO) kindly provided valuable information concerning the VPH gratings. We would also like to thank Bernard Delabre (ESO) for allowing us to adopt the HRS camera design.

\section{References}

Hill, G. J., MacQueen, P. J., Tufts, J. R., Kelz, A., Roth, M. M., Altmann, W., Segura, P., Smith, M., Gebhardt, K., \& Palunas, P. 2006, Proc. SPIE, 6269, 79

Kelz, A., Bauer, S. M., Grupp, F., Hill, G. J., Popow, E., Palunas, P., Roth, M. M., MacQueen, P. J., \& Tripphahn, U. 2006, Proc. SPIE, 6273, 121

Buckley, D. A. H., Cottrell, P. L., Nordsieck, K. H., O’Donoghue, D. E., \& Williams, T. B. 2004, Proc. SPIE, 5492

Barnes, S., Cottrell, P. L., Albrow, M. D., \& Kershaw, G. 2005, 3210AE0005 Optical Design, $03 / 17 / 2005$, Issue 2.7

Davies, R. L., Kuntschner, H., Emsellem, E., Bacon, R., Bureau, M., Carollo, C. M., Copin, Y., Miller, B. W., Monnet, G., Peletier, R. F., Verolme, E. K., \& de Zeeuw, P. T. 2001, ApJ $548,33-36$

Bacon, R., Copin, Y., Monnet, G., Miller, Bryan W., Allington-Smith, J. R., Bureau, M., Carollo, C. M., Davies, R. L., Emsellem, E., Kuntschner, H., Peletier, R. F., Verolme, E. K., \& de Zeeuw, P. T. 2001, MNRAS 326, 23-35 